\title{
Validación de la entrevista diagnóstica estructurada DISC-IV para la identificación de trastornos psiquiátricos en niños y adolescentes en Chile Validation of the DISC-IV structured diagnostic interview to identify psychiatric disorders in children and adolescents in Chile
}

Sandra Saldivia B. ${ }^{1, a}$, Benjamín Vicente P. ${ }^{1}$, Mario Valdivia P. ${ }^{1}$ y Roberto Melipillán A. ${ }^{1, b}$

Introduction: In Chile there are scarce validated instruments in order to perform categorical psychiatric diagnosis both in children and adolescents. DISC-IV Spanish version validation indexes are presented. Method: A convenience sample was collected from outpatient psychiatric services as well as from private medical facilities and drug-dependence outpatient centres from Concepción, Chile. Subjects of both genders between 7 and 18 years of age, with a psychiatric diagnosis were included in the study (affective disorder, anxiety, drugs abuse and dependence, and behaviour disorder). In addition 18 subjects without psychiatric disorder were selected from a secondary school. Each selected subject that agreed to participate in the study was interviewed by a clinical experienced child psychiatrist, diagnose-blinded, using DSM-IV check list, in order to decide if the subject fulfilled DSM-IV criteria. Later, to patients 12 years old or older, or to their caregiver for children younger than 12 years DISC-IV was applied by some of the previously trained lay interviewers. Quality controls of the interviews were carried out. Data were entered to SPSS file to obtain DISC-IV validation indexes. Results: 144 subjects were incorporated in the study. Cohen's Kappa indexes, a statistical measure of inter-rater agreement, varied between 0.53 for anxiety disorders, and 0.88 for substance abuse and dependence. Every group presented high specificity ranging between 0.55 and 0.83. Interviews answered by adolescents were less sensitive for TDA (0.23) and anxiety (0.40), whereas in parents, sensitivity decreases for affective disorders (0.50). Conclusion: DISC-IV is a valid instrument to be used in the research about children and adolescent mental health.

Key words: Diagnostic interview, adolescent, DISC-IV, psychiatric disorder. Rev Chil Neuro-Psiquiat 2013; 51 (1): 70-78

Recibido: $12 / 12 / 2012$

Aceptado: 15/03/2013

Financiamiento: DIUC 206.087.037-1.0.

Departamento de Psiquiatría y Salud Mental, Facultad de Medicina, Universidad de Concepción.

Doctora en Psicología

Psicólogo, Magíster en Investigación Social. 


\section{Introducción}

$\mathrm{U}$ na de los desafíos para la realización de estudios epidemiológicos que permitan conocer la prevalencia de patología psiquiátrica en la población infantil y adolescente del país es poder contar con un instrumento que permita efectuar diagnósticos categoriales en grandes muestras de población y que esté validado en el contexto en que se aplicará. En esta búsqueda, el Instituto de Salud Mental de Estados Unidos (NIMH) impulsó el trabajo denominado Methods for the Epidemiology of Child and Adolescent (MECA) ${ }^{1-5}$, fruto del cual se desarrollaron entrevistas epidemiológicas, una de las cuales es el Diagnostic Interview Schedule for Children (DISC) ${ }^{2}$.

El DISC es una entrevista altamente estructurada diseñada para realizar diagnósticos precisos de acuerdo con los criterios DSM-IV y CIE-10 2, 5-7. Puede ser administrada por entrevistadores legos, sin experiencia clínica pero entrenados en su uso. Dispone de dos formas, una para padres (DISC-P) y otra para niños (DISC-C). La entrevista con el niño puede aplicarse en una sola sesión de una hora de duración; la forma para padres dura entre 60 y 90 min. Da cuenta también del inicio, duración y severidad del trastorno. El DISC ha conseguido imponerse por sobre otras entrevistas debido a la gran cantidad de estudios y los excelentes resultados que de ellos se han obtenido ${ }^{5,7-12}$. Una de las características más importantes que la hacen adecuada para ser validada en Chile es que ha sido traducido al español y probada con población latina $^{6,11,13-15}$.

Desde su creación, el DISC ha sido sometido a un gran número de revisiones con el fin de mejorar su eficiencia, sensibilidad, fiabilidad y validez ${ }^{2,4,12,16-19}$. Costello y cols. $(1984)^{5}$, estudiaron la validez de criterio en una muestra pediátrica y otra psiquiátrica usando ambas entrevistas, DISC-P y DISC-C, mostrando que el $95 \%$ y $98 \%$ de ambas muestras, respectivamente, habían sido correctamente clasificadas en cada grupo de estudio, y las puntuaciones obtenidas por el grupo psiquiátrico fueron sistemáticamente superiores a las del grupo pediátrico. Otra de las ventajas del instrumento es su relativo bajo costo para este tipo investigación, ademas tiene un gran potencial para la estandarización y reducción de errores y cuenta con la capacidad de brindar una exhaustiva y meticulosa búsqueda de psicopatología infantil y adolescente gracias a su formato. Entre las limitaciones se incluyen la poca habilidad para pesquisar respuestas invalidadas proporcionadas por quien no entienda el cuestionario, además de la incapacidad de pesquisar presentaciones atípicas, ya que está diseñado para evaluar los síntomas de acuerdo a las clasificaciones DSM y CIE.

La confiabilidad test-retest del DISC-IV, la última versión del instrumento, ha sido reportada en estudios clínicos tanto para su versión en español como inglesa, con resultados comparables para ambas ${ }^{6,11}$. Además, desde la versión III el DISC es compatible con el sistema de clasificación de DSMIV y CIE-10.

Dada las características psicométricas del DISC IV, su amplia utilización en estudios internacionales y la existencia de una versión en español, el instrumento resultaba potencialmente adecuado para su utilización en la población infanto-adolescente de Chile. Se presentan los índices de validación obtenidos de la versión en español del DISC-IV.

\section{Método}

Estudio descriptivo para estudiar la confiabilidad de cuatro de los 6 módulos diagnósticos del DISC-IV, versión computarizada: trastornos ansiosos, trastornos del ánimo, trastornos por abuso de sustancias y trastorno de conducta. Se exceptuó Misceláneos y Esquizofrenia, por las dificultades para encontrar casos en patologías de baja prevalencia como trastorno de eliminación, pica, bulimia o esquizofrenia.

\section{Muestra}

Se seleccionaron 18 sujetos, de ambos sexos, de entre 4 y 18 años, por cada uno de los 4 módulos diagnósticos, y 18 sujetos sin diagnóstico psiquiátrico. Se excluyeron casos con retardo mental profundo o moderado. La muestra fue recogida del centro de atención ambulatoria del Servicio de 
Psiquiatría del Hospital Clínico Regional de Concepción, de la Unidad de Salud Mental del Hospital Las Higueras de Talcahuano, algunos pacientes de consultas privadas y, para el caso drogodependencias, de centros de tratamiento especializados, públicos y/o con financiamiento estatal.

\section{Instrumentos y medidas}

La Entrevista Diagnóstica para Niños (DISC) es un instrumento de diagnóstico altamente estructurado, diseñado para su uso por no clínicos. El instrumento ha estado en desarrollo desde 1979 y se han producido diversas versiones para calzar con diferentes sistemas de clasificación. La versión actual del DISC (Instituto Nacional de Salud Mental [NIMH] DISC-IV), basado en el DSM-IV y la CIE-10, fue lanzada para su uso en terreno en 1997 y tiene una única versión informatizada. Existen versiones paralelas del instrumento: el DISC-P para padres (o cuidadores con conocimiento) de hijos entre los 6 y 17 años de edad, y el DISC-Y (para su directa administración en niños o jóvenes entre los 9 y 17 años de edad). La información de estas entrevistas puede ser combinada o examinada por separado.

El proceso de validación de la entrevista usó como base la última traducción al español del DISC-IV ${ }^{11}$, realizada por investigadores de Puerto Rico y que cuenta con versiones de entrevistas paralelas para jóvenes y padres. La confiabilidad del testretest de esta versión ha sido informada tanto en muestras clínicas para hispano-parlantes como para anglo-parlantes, con resultados comparables ${ }^{6,11}$.

El DISC-IV también indaga sobre el nivel de discapacidad y severidad asociado con cada diagnóstico, a través de sondeos que determinan el grado en el cual los síntomas de un diagnóstico dado han causado estrés al niño, o han afectado su rendimiento escolar, o sus relaciones con cuidadores, familia, amigos o profesores. La confiabilidad del test-retest respecto de los sondeos sobre discapacidad y severidad fueron de aceptables a moderado; los valores $k$ estuvieron en un rango de 0,42 a 0,80 para la mayoría de los desórdenes evaluados ${ }^{11}$.

Siguiendo la sugerencia de investigadores que han llevado a cabo estudios usando el DISC, los niños menores de 12 años no fueron entrevistados directamente y la información se recogió de sus padres, porque hay evidencia en el sentido que sus informes no serían confiables ${ }^{17}$. Asimismo, se entrevistó directamente a los adolescentes entre 12 y 18 años de edad, pero no a sus padres, dado que éstos tienden a no tener conciencia de algunas conductas más desadaptativas de sus hijos.

Los algoritmos de puntaje oficial del DISC-IV permiten la comprobación de la presencia de un diagnóstico, con o sin discapacidad, según ha sido medido por las escalas de discapacidad de la propia entrevista, a la vez que permite identificar casos sub-umbrales. Para efecto diagnóstico, un caso es considerado positivo si cumple completamente los criterios de diagnóstico del DSM-IV o CIE-10, de acuerdo con el DISC-IV.

Entrenamiento y control de calidad. El proceso se realizó con 6 entrevistadores legos, que, sin embargo, tenían experiencia en la aplicación de entrevistas estructuradas, dado que habían participado en estudios previos y estaban capacitados en la Composite International Diagnostic Interview $(\mathrm{CIDI})^{20}$, entrevista ampliamente utilizada en estudios epidemiológicos en población adulta y cuya estructura es muy similar a la del DISC-IV. Estos entrevistadores, con formación técnica y/o estudiantes universitarios, recibieron un curso de formación de 40 horas con una práctica intensiva con sujetos normales y pacientes, simulados y reales. Todas las entrevistas fueron grabadas en audio, siendo posteriormente revisadas por uno de los investigadores que no participaba del trabajo de campo, a fin de asegurar que fueron completadas adecuadamente. Cuando surgieron problemas en la revisión, estos fueron discutidos con el entrevistador, lo que permitió resolverlos, pero, además, facilito un mejoramiento continuo de la calidad. El coordinador del trabajo de campo visito al azar uno de cada 10 sujetos entrevistados, para determinar que efectivamente el proceso fue realizado de manera adecuada.

\section{Procedimiento}

Adquirida la licencia para el uso del software, la entrevista fue revisada por el equipo investigador 
y se realizó un proceso de adecuación lingüística, el que fue sometido a un pilotaje en una muestra no clínica, lo que dio como resultado la identificación y adecuación de las principales diferencias detectadas.

La identificación de los casos se inició con su identificación en las consultas clínicas. Identificado el paciente, éste y su cuidador fueron invitados a participar del estudio. Si aceptaban, se les citaba a una entrevista realizada por psiquiatras infantiles con experiencia clínica, ciegos al diagnóstico del paciente, y quienes definieron si éste cumplía con los criterios clínicos del DSM-IV para el diagnóstico respectivo.

Si el diagnóstico inicial era confirmado se procedía a aplicar la versión computarizada del DISCIV-Y, cuando el paciente tenía 12 años o más, o el DISC-P, aplicada a uno de los padres o cuidador principal, para los menores de 12 años; además del módulo de información general e historia de vida. Las entrevistas se realizaron "cara a cara" en el hogar del entrevistado o en instalaciones universitarias, de acuerdo a la decisión del paciente y/o de su cuidador. Al inicio, el entrevistador, quien es lego respecto del diagnóstico clínico del paciente, solicitaba la firma del formulario de consentimiento informado del adolescente entrevistado y/o del adulto responsable, y entonces procedía a aplicar la entrevista.

Los entrevistados sin diagnostico psiquiátrico fueron contactados en un establecimiento educacional y en la comunidad, siendo sometidos al mismo procedimiento ya descrito.

\section{Manejo de datos}

Como se ha mencionado, la entrevista DISC-IV permite obtener el o los diagnostico(s) clínico(s) para cada paciente, de acuerdo a criterios DSMIV o CIE-10; y los resultados de los algoritmos de discapacidad. Para este proceso se utilizaron los criterios diagnósticos DSM-IV, los que permitieron obtener los índices de validación para cada módulo estudiado.

\section{Resultados}

Un total de 144 sujetos fueron ingresados al estudio. De ellos, la mayor proporción presentaba un cuadro afectivo $(22,2 \%)$. Fue menos frecuente la confirmación diagnóstica de los trastornos de ansiedad, por lo cual solo se ingresaron 15 casos. El módulo de trastornos de conducta fue dividido en dos grupos, por un lado, los trastornos de conducta propiamente tales y el trastorno oposicionista desafiante, y por otro, el trastorno por déficit atencional, considerando la mayor frecuencia con que este último cuadro se presenta en la consulta clínica y las diferencias en la psicopatología de ambos grupos (Tabla 1).

En el conjunto de la muestra los hombres son más frecuentes, lo que se repite para la sub muestra de los módulos de abuso y dependencia y de trastornos de conducta, incluyendo el trastorno por déficit atencional. En sentido inverso, había más mujeres en el grupo de pacientes identificadas con cuadros afectivos y ansiosos. El grupo de pacientes

Tabla 1. Descripción de la muestra

\begin{tabular}{|c|c|c|c|c|c|c|c|}
\hline & Afectivos & Ansiosos & $\begin{array}{c}\text { Abuso y } \\
\text { dependencia }\end{array}$ & $\begin{array}{l}\mathrm{TC}^{1}+ \\
\mathrm{TOD}^{2}\end{array}$ & $\mathrm{TDA}^{3}$ & Sanos & Total \\
\hline Total de la muestra & $32(22,2 \%)$ & $15(10,4 \%)$ & $23(16,0 \%)$ & $32(22,2 \%)$ & $24(16,7 \%)$ & $18(12,5 \%)$ & $144(100 \%)$ \\
\hline Masculino & $14(43,8 \%)$ & $6(40,0 \%)$ & $19(82,6 \%)$ & $26(81,3 \%)$ & $21(87,5 \%)$ & $16(88,9 \%)$ & $102(70,8 \%)$ \\
\hline Media de edad (sd) & $13,9 \quad(3,7)$ & $11,4 \quad(2,2)$ & $16,6 \quad(1,6)$ & $12,7 \quad(3,3)$ & $10,7 \quad(3,2)$ & $14,4 \quad(3,0)$ & $13,3 \quad(3,5)$ \\
\hline \multicolumn{8}{|l|}{ Grupo etario } \\
\hline 8 - 11 años & $9(19,1 \%)$ & $9(19,1 \%)$ & - & $11(23,4 \%)$ & $16(34,0 \%)$ & $2(4,3 \%)$ & $47(32,6 \%)$ \\
\hline $12-18$ años & $23(23,7 \%)$ & $6(6,2 \%)$ & $23(23,7 \%)$ & $21(21,6 \%)$ & $8 \quad(8,2 \%)$ & $16(16,5 \%)$ & $97(67,4 \%)$ \\
\hline
\end{tabular}

${ }^{1}$ TC: Trastorno de conducta; ${ }^{2}$ TOD: Trastorno oposicionista-desafiante; ${ }^{3}$ Trastorno por déficit atencional. 
Tabla 2. Resultados psicométricos para los módulos validados

\begin{tabular}{|lccccc|}
\hline & Afectivos & Ansiosos & Abuso y dependencia & $\begin{array}{c}\text { Trastornos de } \\
\text { conducta + TOD }\end{array}$ & TDA \\
Sensibilidad & 0,65 & 0,55 & 0,83 & 0,75 & 0,60 \\
Especificidad & 1,00 & 0,94 & 1,00 & 1,00 & 1,00 \\
VP+ & 1,00 & 0,86 & 1,00 & 1,00 & 1,00 \\
VP- & 0,75 & 0,77 & 0,95 & 0,90 & 0,75 \\
Índice de concordancia & 0,83 & 0,79 & 0,96 & 0,92 & 0,82 \\
KAPPA & 0,65 & 0,53 & 0,88 & 0,81 & 0,62 \\
\hline
\end{tabular}

con cuadros ansiosos y con trastornos por déficit atencional incluyen, en promedio, más niños; mientras que la muestra de pacientes que presentan abuso y dependencia estuvo conformada sólo por adolescentes (Tabla 1).

Los resultados de la validación del instrumento para el total de la muestra, considerando el juicio clínico como patrón de referencia (gold standard), se presentan en la Tabla 2. Los valores del Índice Kappa de Cohen son, de acuerdo al criterio de Fleiss, excelente para abuso y dependencia, trastornos de conducta y oposicionista desafiante (Kappa > ,75); y bueno para los trastornos afectivos, los cuadros ansiosos y el déficit atencional $(, 40<$ Kappa $<, 75)$.

En todos los casos, la especificidad es alta, con una elevada probabilidad de diagnosticar como sanos a aquellos que efectivamente lo son; pero se observan importantes variaciones en los valores de sensibilidad de los diferentes módulos, siendo más baja para los trastornos ansiosos, con lo cual estos cuadros podrían ser sub-diagnosticados por el DISC-IV.

Si se revisan los resultados de acuerdo al criterio de quien ha respondido a la entrevista, o los padres o los adolescentes, se observan interesantes variaciones (Tabla 3). La sensibilidad en el módulo de cuadros afectivos es mayor entre los adolescentes, quienes tienden a reportar
Tabla 3. Resultados psicométricos para los módulos validados, por grupo etario. Módulos para padres

\begin{tabular}{|lcccc|}
\hline & Afectivos & Ansiosos & $\begin{array}{c}\text { Trastornos de } \\
\text { conducta + TOD }\end{array}$ & TDA \\
\hline Sensibilidad & 0,50 & 0,67 & 1,00 & 0,89 \\
Especificidad & 1,00 & 1,00 & 1,00 & 1,00 \\
VP+ & 1,00 & 1,00 & 1,00 & 1,00 \\
VP- & 0,50 & 0,50 & 1,00 & 0,67 \\
Índice de & & & & \\
concordancia & 0,67 & 0,75 & 1,00 & 0,91 \\
KAPPA & 0,40 & 0,50 & 1,00 & 0,74 \\
\hline
\end{tabular}

Tabla 4. Resultados psicométricos para los módulos validados, por grupo etario. Módulos para adolescentes

\begin{tabular}{|lcccc|}
\hline & Afectivos & Ansiosos & $\begin{array}{c}\text { Trastornos de } \\
\text { conducta + TOD }\end{array}$ & TDA \\
Sensibilidad & 0,69 & 0,40 & 0,67 & 0,17 \\
Especificidad & 1,00 & 0,94 & 1,00 & 1,00 \\
VP+ & 1,00 & 0,67 & 1,00 & 1,00 \\
VP- & 0,80 & 0,83 & 0,89 & 0,76 \\
Índice de & & & & \\
concordancia & 0,86 & 0,81 & 0,91 & 0,77 \\
KAPPA & 0,71 & 0,40 & 0,74 & 0,23 \\
\hline
\end{tabular}

más precisamente su sintomatología (Tabla 4). Un resultado inverso se obtiene en el trastorno por déficit atencional, cuyos síntomas son menos reportados por los adolescentes de lo que clínicamente fue diagnosticado, disminuyendo notoriamente la sensibilidad del instrumento. En la misma línea están los trastornos de conducta y el trastorno oposicionista desafiante, aunque aquí la sensibilidad es más alta y el índice 
de concordancia elevado en ambos grupos. Finalmente, los trastornos ansiosos son sub-diagnosticado en ambos grupos, aunque este sub diagnóstico es mayor en el grupo de los adolescentes.

\section{Discusión}

En Chile la investigación en epidemiología psiquiátrica infantil y adolescente es escasa y una razón es la inexistencia de instrumentos validados que permitan hacer diagnósticos en forma confiable, lo que es considerado un requisito para la investigación en este ámbito. Este estudio busca dar una respuesta a este déficit, validando un instrumento ampliamente usado, principalmente en países desarrollados, para realizar diagnóstico psicopatológico en niños y adolescentes tempranos. Sin embargo, y aunque existe una versión en español que se generó en Puerto Rico que ha mostrado ser confiable y válida ${ }^{14}$, su uso en América Latina ha sido limitado.

Si bien los resultados reportados respaldan el uso de la entrevista DISC-IV en la poblacion de niños y adolescentes de Chile, el estudio presenta algunas limitaciones. La primera refiere a los resultados obtenidos en el modulo de trastornos ansiosos, cuyo diagnostico podría ser subvalorado. En este caso, y considerando el uso de la entrevista en poblaciones más amplias, podría ser necesario complementar la entrevista con algún cuestionario que fuera más sensible a la presencia de este tipo de cuadros, o - en el caso de estudios clínicos - confirmar la presencia/ausencia del diagnóstico con una evaluación en profundidad. La segunda limitación refiere al uso de los criterios de discapacidad frecuentemente usados en estudios con menores y adolescentes. Los resultados aquí reportados incluyen únicamente el diagnostico clínico, pero los estudios epidemiológicos en esta población sugieren la importancia de sumar a éste una evaluación de la discapacidad asociada a cada cuadro, dada la tendencia a sobre diagnosticar si se utilizan sólo criterios clínicos. Realizar tal procedimiento requiere muestras más grandes de pacientes.

Los resultados del estudio muestran índices de validación del DISC-IV comparables o aún superiores a los obtenidos por Canino y Bird (1987) $)^{14}$ en el estudio de validación en Puerto Rico (Kappa $>, 4$ ), excepto para el trastorno por déficit atencional medido en los adolescentes.

Siguiendo la sugerencia de investigadores que han llevado a cabo estudios usando el DISC, los niños menores de 12 años no fueron entrevistados directamente y la información se recogió de sus padres, porque hay evidencia en el sentido que sus informes no serían confiables ${ }^{17}$. En esta misma línea pueden estar los resultados encontrados para el trastorno por déficit atencional, donde el reporte de los padres es más preciso y ajustado al gold standard que el de los adolescentes, lo que puede estar asociado al egocentrismo propio de este periodo que impide valorar las conductas desajustadas como síntomas de un diagnostico clínico.

Por otra parte, las entrevistas sobre trastornos por consumo de substancias fueron administradas a los púberes y adolescentes entre los 12 y 18 años de edad, pero no a sus padres, dado que éstos tienden a no tener conciencia del uso de substancias por parte de sus hijos adolescentes y no son considerados como fuentes confiables de información para este tipo de desórdenes. Los resultados de la validación de este módulo muestran adecuados resultados y parecen apoyar esta decisión. Se observa además una importante dificultad para encontrar casos clínicos de abuso y dependencia en menores de 12 años.

En un sentido inverso, los padres son menos precisos en la identificación de síntomas afectivos en sus hijos pequeños, que los adolescentes respecto de los propios. El resultado puede estar marcado por una tendencia a la introspección que se incrementa en la adolescencia, pero también alerta acerca de la necesidad de educar a los padres en el conocimiento y reconocimiento de signos y síntomas que pueda esconder la presencia de un cuadro afectivo.

La identificación de trastornos de conducta y del trastorno oposicionista desafiante alcanza buenos resultados tanto en la muestra general como en la de cada grupo por separado. Es probable que a la base de este buen redimiento de la prueba estén las 
características clínicas de los cuadros, incluyendo los aspectos de conducta disrruptiva, que los hacen mas visibles, no necesariamente como un diagnóstico clínico, pero si como para ser reportados cuando se indaga acerca de ellos.

La mayor probabilidad de sub-diagnosticar cuadros ansiosos podría estar asociada a una mayor presencia de cuadros sub-umbrales, por una parte, $y$, por otra, por la co-morbilidad que fue más frecuente en esta sub-muestra de pacientes, con presencia de cuadros afectivos, deficit atencional y trastornos conductuales. Desde esta perspectiva, cabe preguntarse una vez más si las categorías diagnósticas del DSM-IV no esconden fenomenos psicopatologicos mas complejos, probablemente dimensionales, que dificultan -en la clínica realhacer separaciones tan claras entre distintos grupos diagnosticos. Si este tema se discute a nivel de la poblacion adulta, con mayor probabilidad puede ser un ámbito de análisis y controversia en el ámbito menos estudiado de la psicopatología infantil $\mathrm{y}$ adolescente.

\section{Agradecimientos}

A J. Cofré, MI. Condeza, C. Llorente, P. Pandolfi y M. Zúñiga, Hospital de Día de Lirquén y CRESER Concepción.

\section{Resumen}

Introducción: En Chile existen escasos instrumentos validados para efectuar diagnósticos psiquiátricos categoriales en población infantil y adolescente. Se presentan los índices de validación obtenidos para la versión en español del DISC-IV. Método: Una muestra por conveniencia fue seleccionada de los registros clínicos de los servicios de psiquiatría ambulatorios de Concepción, consultas privadas y centros de atención a drogodependientes. Ésta incluyó a sujetos de ambos sexos, con edades entre 7 y 18 años, que presentaran algún diagnóstico incluido en el estudio (trastornos del ánimo, ansiosos, abuso y dependencia a sustancias y trastornos del comportamiento). Además se seleccionaron de un establecimiento educacional a 18 sujetos sin diagnóstico psiquiátrico. Cada sujeto seleccionado que aceptó participar del estudio, y el cuidador responsable, fueron entrevistados por un psiquiatra infantil entrenado, ciego al diagnóstico inicial, utilizando un check-list basado en los criterios DSM-IV, para obtener diagnósticos categoriales. Posteriormente, entrevistadores legos previamente capacitados, aplicaron al paciente, si tenía 12 años o más, o a su cuidador principal, para los menores de 12 años, la entrevista estructurada DISC-IV. Se realizaron controles de calidad de las entrevistas. Los datos fueron ingresados a un archivo SPSS para ser procesados y obtener índices de validación del DISC-IV. Resultados: Ciento cuarenta y cuatro sujetos ingresaron al estudio. Los Índice Kappa de Cohen, que miden el acuerdo inter-evaluadores, varían entre,53, para los trastornos ansiosos, y,88, para abuso y dependencia. En todos los grupos la especificidad es alta $(>0,9)$ y la sensibilidad varía entre , 55 y,83. Las entrevistas respondidas por adolescentes son menos sensibles para TDA $(0,23)$ y ansiedad $(0,40)$, mientras en los padres la sensibilidad disminuye para los cuadros afectivos $(0,50)$. Conclusión: El DISC-IV es un instrumento válido para ser usado en la investigación en salud mental infanto-adolescente.

Palabras clave: DISC-IV, entrevista diagnóstica, infantil, adolescente, trastorno psiquiátrico. 


\section{Referencias bibliográficas}

1. Lohey BB, Flagg EW, Bird HR, Li OL. The NIMA Methods for the Epidemiology of Child and Adolescent Mental Disorders (MECA) Study: background and methodology. J Am Acad Child Adolesc Psychiatry 1996; 35: 855-64.

2. Shaffer D, Schwab-Stone M, Fisher P, Cohen P, Piacentini J, Davies M, et al. The Diagnostic Interview Schedule for Children-Revised Version (DISC-R): I. Preparation, field testing, interrater reliability, and acceptability. J Am Acad Child Adolesc Psychiatry 1993; 32: 643-50.

3. Shaffer D, Fisher P, Dulcan MK, Davies M, Piacentini J, Schwab-Stone ME, et al. The NIMH Diagnostic Interview Schedule for Children Version 2.3 (DISC-2.3): description, acceptability, prevalence rates and performance in the MECA Study: Methods for the Epidemiology of Child and Adolescent Mental Disorders Study. J Am Acad Child Adolesc Psychiatry 1996; 35: 865-77.

4. Schwab-Stone M, Shaffer D. Criterion Validity of the NIMH Diagnostic Interview Schedule for Children Version 2.3 (DISC-2.3). J Am Acad Child Adolesc Psychiatry 1996; 35: 878-88.

5. Costello AJ, Edelbrock CS, Dulcan MK, Kalas R, Klaric SH. Development and testing of the NIMH Diagnostic Interview for children in a clinic population. Final Report. Center for Epidemiologic Studies, National Institute of Mental Health. Rockville, MD. 1984.

6. Shaffer D, Fisher P, Lucas C, Dulcan M, SchwabStone M. NIMH Diagnostic Interview Schedule for Children Version IV (NIMH DISC-IV): Description, differences from previous versions and reliability of some common diagnoses. J Am Acad Child Adolesc Psychiatry 2000; 39: 28-38.

7. Cutterman EM, O’Brian JD, Young JG. Structured diagnostic interviews for children and adolescent: Current status and future directions. J Am Acad Child Adolesc Psychiatry 1987; 26: 62-630.

8. Bird H, Gould M, Stghezza B. Aggregating data from multiples informants in child psychiatry epidemiological research. J Am Acad Child Adolesc Psychiatry 1992; 311: 78-85.

9. Leaft PJ, Alegria M, Cohen P, Goodman SH,
Horwitz SM, Hoven CW, et al. Mental health service use in the community and schools: results from the Four-Community MECA Study. Methods for the Epidemiology of Child and Adolescent Mental Disorders Study. J Am Acad Child Adolesc Psychiatry 1996; 35: 889-97.

10. Costello EJ, Angold A, Burns BJ, Stangl DK, Tweed DL, Erkanli A, et al. The Great Smoky Mountains Study of Youth: goals, design, methods, and the prevalence of DSM-III-R disorders. Arch Gen Psychiatry 1996; 53: 1129-36.

11. Bravo M, Ribera J, Rubio-Stipec M, Canino G, Shrout P, Ramírez R, et al. Test-retest reliability of the Spanish version of the Diagnostic Interview Schedule for Children (DISC-IV). J Abnorm Child Psychol 2001; 29: 433-44.

12. Lucas C, Zhang H, Fisher PW, Shaffer D, Regier DA, Narrow WE, et al. The DISC Predictive Scales (DPS): Efficiently screening for diagnoses. J Am Acad Child Adolesc Psychiatry 2001; 40: 443-9.

13. Karno M, Burman A, Escobar J, Hough R, Eaton W. Development of the Spanish-Language Version of the National Institute of Mental Health Diagnostic Interview Schedule. Arch Gen Psychiatry 1983; 40: 1183-8.

14. Canino G, Bird H, Shrout PE, Rubio-Stipec M, Bravo M, Martinez R, et al. The Spanish Diagnostic Interview Schedule: Reliability and concordance with clinical diagnosis in Puerto Rico. Arch Gen Psychiatry 1987; 44: 720-6.

15. Canino G, Shrout PE, Rubio-Stipec M, Bird HR, Bravo M, Ramírez R, et al. The DSM-IV rates of child and adolescent disorders in Puerto Rico. Prevalence, correlates, service use, and the effects of impairment. Arch Gen Psychiatry 2004; 6185-93.

16. Fisher PW, Shaffer D, Piacentini JC, Lapkin J, Kafantaris V, Leonard H, et al. Sensitivity of the Diagnostic Interview Schedule for Children, 2nd edition (DISC-2.1) for specific diagnoses of children and adolescents. J Am Acad Child Adolesc Psychiatry 1993; 32: 66-673.

17. Schwab-Stone M, Fallon T, Briggs M, Crowther B. Reliability of diagnostic reporting for children aged 6-11 years: a test-retest study of the Diagnostic Interview Schedule for Children-Revised. Am J Psychiatry 1994; 151: 1048-54. 
18. Jensen P, Rubio-Stipec M, Canino G, Bird H, Dulcan M, Schwab-Stone M, et al. Parent and child contributions to diagnosis of mental disorder: Are both informants always necessary? J Am Acad Child Adolesc Psychiatry 1999; 38: 1569-79.

19. Hough RL. Comparing DISC-IV and clinician Diagnoses among youths receiving public mental health services. J Am Acad Child Adolesc Psychiatry 2003; 42: 349-56.

20. Robins LN, Wing J, Wittchen HU, Helzer JE, Babor TF, Burke J, et al. The Composite International Diagnostic Interview: an epidemiologic instrument suitable for use in conjunction with different diagnostic systems and in different cultures. Arch Gen Psychiatry. 1988; 45: 1069-77.

Correspondencia:

\section{Sandra Saldivia B.}

Departamento de Psiquiatría y Salud Mental, Facultad de Medicina, Universidad de Concepción. Casilla 160-C, Concepción, Chile.

Teléfono: 41-231 2799

E-mail: ssaldivi@udec.cl 